\title{
CANCIONES ACRÍTICAS Y LAMENTOS FÚNEBRES. INTERRELACIONES EN LA REGIÓN DE TRACIA
}

\author{
Ana Isabel Fernández Galvín \\ Centro de Estudios Bizantinos, Neogriegos y Chipriotas (Granada) \\ catulo1@gmail.com
}

\section{RESUMEN}

Algunas canciones acríticas continúan aún vivas en el pueblo griego, adaptándose a las nuevas circunstancias, consecuencia de la plurifuncionalidad de la canción popular. Incluso pueden entonarse en los funerales. Ello ha llevado a la inclusión de este tipo de composiciones dentro de la categoría de los miroloyia o de las canciones de Jaros, con las que comparten motivos, temas y versos formularios.

En el presente artículo se analizan las interrelaciones entre estas categorías de canciones en la región de Tracia, extrapolables al resto del territorio heleno.

PALABRAS CLAVE: Canción popular neogriega, canciones acríticas, miroloyia, canciones de Jaros, Tracia.

\section{ABSTRACT}

«Acritic and mourning songs. Interrelationships in Thrace». Some acritic songs are still alive in the Greek people, adapting to new circumstances, due to the multifunctionality of the folk song. Even they can be sung at funerals. It has led to the inclusion of these compositions within the categories of mirológia or moirológia of the Underworld and Charos, with which they share themes, motives and verses forms.

This study focuses on the interrelationships between these song categories in Thrace, that can be extrapolated to the rest of the Greek territory.

KEY WORDS: Modern Greek folk songs, acritic songs, moirológia, moirológia of the Underworld and Charos, Thrace.

Integradas antropológicamente en los llamados ritos de paso ${ }^{1}$, las canciones populares de muerte se clasifican en miroloyia $\left(\mu \circ \varrho o \lambda \dot{o}_{\gamma} \alpha^{2}\right)$ y canciones de Jaros

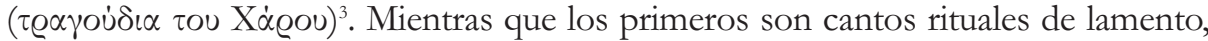
a modo de treno, por la muerte de una persona amada, en los que se destacan sus virtudes y, a veces, parte de su biografía, o simplemente canciones tristes en boca del propio difunto o sus familiares, lamentando la pérdida de la vida y las penalidades del Mundo Inferior ${ }^{4}$, los segundos constituyen un ciclo temático de alegorías tradicionales sobre el Hades, basadas en una personificación, de origen medieval, entrecruzadas con temas de la poesía acrítica. No están relacionadas con casos concretos, 
sino que elevan, en un nivel más general, a la categoría de mito el enfrentamiento entre la vida y la muerte por medio de la personificación de las fuerzas contendientes.

Las canciones acríticas (s. VIII-XIII), vinculadas a algunos episodios del poema épico Diyenís Acritas o que celebraban a los defensores de las fronteras orientales del Imperio Bizantino, con el cambio de las condiciones sociales y militares, dejaron de componerse, aunque han continuado vivas en el pueblo griego hasta nuestros días. Por eso han sufrido grandes transformaciones, adaptándose a las nuevas circunstancias, prueba de la plurifuncionalidad de la lírica popular. Así en algunas composiciones fúnebres en las que el difunto relata sus hazañas puede rastrearse el conocido pasaje en el que Diyenís, en su lecho de muerte, cuenta a sus amigos sus proezas. Además determinadas formas derivadas de la tradición acrítica sobre la muerte del héroe épico, que reproducen con gran libertad algunos episodios de sus gestas, se cantan como miroloyia ${ }^{6}$. Estas canciones, recogidas ya en las recopilaciones más antiguas $^{7}$, con un interés especial no solo por su cantidad ${ }^{8}$ sino también por su calidad, fusionan elementos perfectamente documentados con otros nuevos, que tienen a menudo su origen en tradiciones locales.

A pesar de que nuestro análisis se centrará en una zona muy concreta del territorio históricamente habitado por los griegos, como Tracia, región con un particular interés etnológico y musical, cruce y mezcla de Oriente y Occidente, con una especial tradición cultural y unas características distintas al resto de Grecia en cuanto a su música y sus bailes, los resultados son extrapolables al resto del territorio helénico.

Algunas composiciones, originariamente acríticas, han sido consideradas ocasionalmente como canciones de muerte, siguiendo una concepción amplia del término. Los dos ejemplos presentados se deben al mismo recopilador tracio y constituyen, en ambos casos, formas muy evolucionadas del prototipo primitivo.

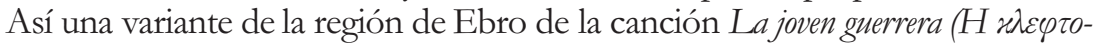

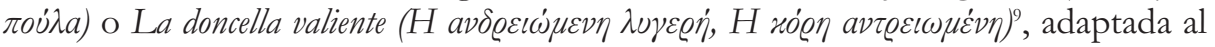
contexto social y local, fue clasificada como canción de Jaros.

${ }^{1}$ Van Gennep, 1909: 185-187 y 209-210; Danford y Tsiaras, 1982: 35-36.

${ }^{2}$ Para un estudio diacrónico del término $c f$. Alexiou, 1974: 102-118.

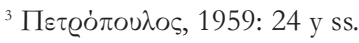

${ }^{4}$ «Carmina composita a feminis mortem propinqui lugentibus», Passow, 1860: 257.

${ }^{5}$ «Quibus Charon omnes mortales superans describitur», Passow, 1860: 291.

${ }^{6}$ Estas son algunas composiciones tracias que narran su muerte: $\sum \tau \alpha \mu \circ \dot{\lambda} \lambda \eta \Sigma \alpha \propto \nu \tau \dot{\eta}, 1929$ :

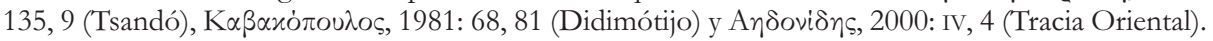
Otras se refieren de forma exclusiva a sus hazañas [ $\sum \tau \alpha \mu o \dot{\lambda} \lambda \eta \sum \alpha \varrho \alpha \nu \tau \dot{\eta}, 1929: 136,1$ (Tsandó) y 1939: 199, 336 (Kastaniés)].

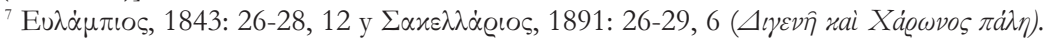

${ }^{8}$ En un estudio del año 2004 se documentaron más de quinientos textos en todo el territorio griego, cf. Ayensa Prat, 2004: 47.

${ }_{9}$ El tema, que aparece desde las primeras recopilaciones [Tommaseo, 1842: 78-84, Euג $\dot{\alpha} \mu \pi \iota$,

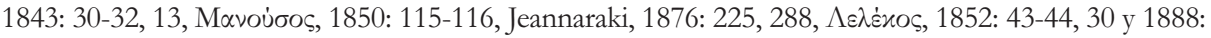




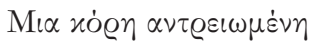

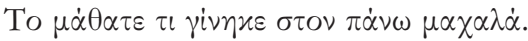

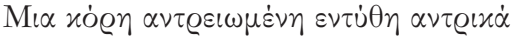

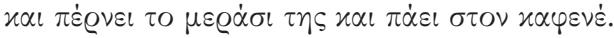

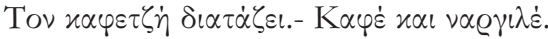

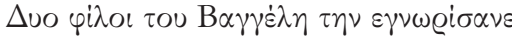

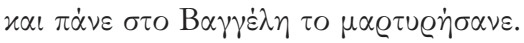

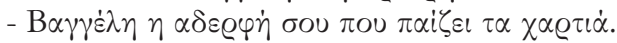

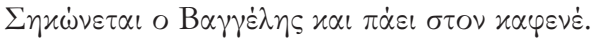

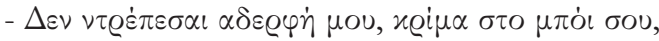

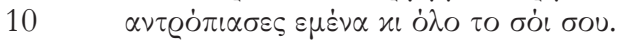

A $\pi^{\prime} \tau \alpha \mu \alpha \lambda \lambda i \dot{\alpha} \tau \eta \nu \pi i \dot{\alpha} \nu \varepsilon l \tau \eta \beta$

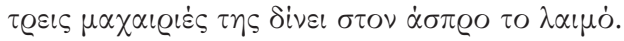

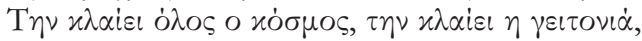

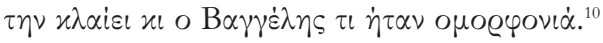

Una doncella valiente

Sabed lo que sucedió en el barrio de arriba.

Una doncella valiente se vistió de hombre, coge y se marcha al café.

Al dueño del café le pide café y narguile.

Dos amigos de Vanguelis la reconocieron, van a Vanguelis y lo denuncian.

- Vanguelis, tu hermana está jugando a las cartas.

Se levanta Vanguelis y va al café.

- ¿No te da vergüenza, hermana mía? Desgraciada seas,

me deshonraste a mí y a todo tu linaje.

La coge por los pelos, la lleva al patio,

tres puñaladas le da en su blanco cuello.

La llora todo el mundo, la llora el barrio,

la llora también Vanguelis por lo bella que era.

Igualmente otra variante muy transformada de la canción acrítica Los hijos

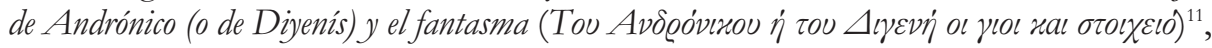
ha sido también incluida en la misma categoría por el anterior recopilador. Presenta,

I, 25-26 y K@ì்ns, 1920: 220-221, 2], entronca con el de las amazonas clásicas, pero parece provenir de las historias del ciclo de Alejandro Magno [Saunier, 1989: 71]. Cf. una versión de Tracia Oriental en M $\pi \dot{\alpha} \varrho \mu \pi \alpha \varsigma, 1996: 28$ (Kalípolis) y dos variantes, del Epiro y de las Cícladas, seguidas de un análisis sobre la canción en Ayensa Prat, 2004: 56-59 y 237-242.

${ }^{10}$ Maveíitทs, 2000: 124, 1.

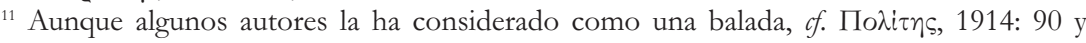
M $\pi \dot{\alpha} \varrho \mu \pi \alpha \varsigma, 1996: 36-37$ (Mega Voyaliki), desde S. Baud-Bovy (1936: 285) ha sido incluida entre las

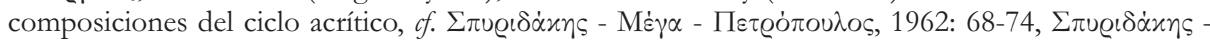

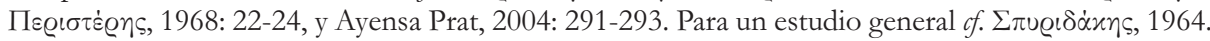
Se encuentra ya en las primeras colecciones, $c f . \Lambda \varepsilon \lambda$ Éxos, 1852: 41-42, 28. 
a modo conclusivo y adaptado a la zona, el motivo metafórico de la boda mítica ${ }^{12}$, propio de los miroloyia dedicados a jóvenes muertos prematuramente antes de sus nupcias.

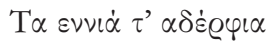

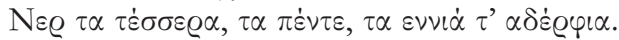

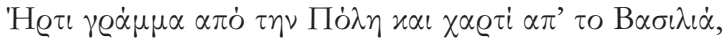

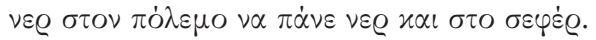

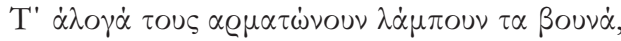

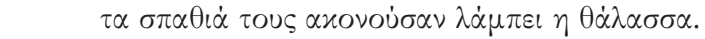

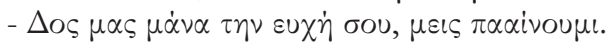

- ' $\Omega \varrho \alpha x \alpha \lambda \dot{\eta} \pi \alpha \iota \dot{\alpha} x \iota \alpha \mu o v, \nu \alpha \pi \dot{\alpha} \tau \varepsilon \sigma \tau \dot{o} x \alpha \lambda \dot{o}$,

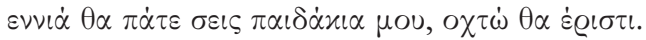

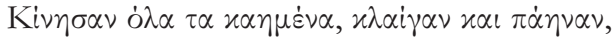

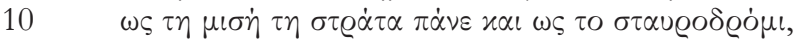

$\delta i \psi \alpha \sigma \alpha \nu$ ò $\lambda \alpha \tau \alpha \pi \alpha \iota \delta i \dot{\alpha}, \nu \varepsilon \varrho o \dot{~} \delta \varepsilon \beta \varrho \dot{\theta} \theta \eta x \varepsilon$.

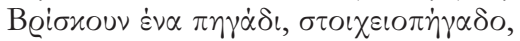

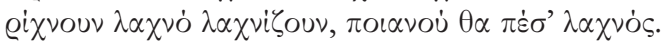

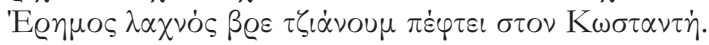

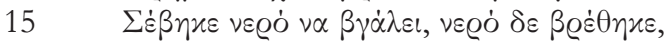

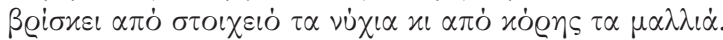

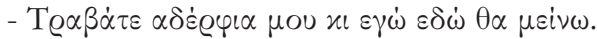

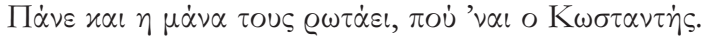

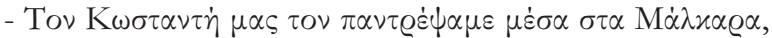

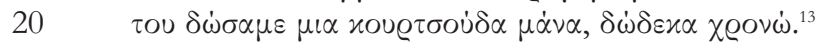

Los nueve hermanos

Los cuatro, los cinco, los nueve hermanos.

Llegó una carta de la Ciudad y un escrito del Emperador, que fueran a la guerra y al ejército.

Sus caballos arman, refulgen las montañas,

5 sus espadas afilaron, refulge el mar.

- Danos, madre, tu bendición, nosotros partimos.

- En buena hora, niños míos, que os vaya bien, nueve os marcháis, niños míos, ocho volveréis.

Se pusieron en marcha todos los desgraciados, al irse lloraban,

10 llegan hasta la mitad del camino, hasta la encrucijada,

les acució la sed a todos, pero agua no se encontró.

Encuentran un pozo, un pozo encantado,

lo echan a suerte, sortean, a quién le tocará.

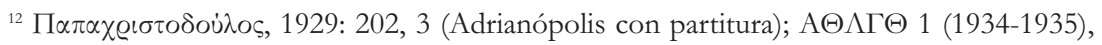

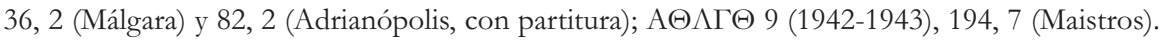

${ }^{13}$ Mav@íंทs, 2000: 120. 
La mala suerte, ay, alma mía, le toca a Costandís.

Entró a sacar agua, pero agua no se encontró,

encuentra las uñas de un fantasma y los cabellos de una doncella.

- Tirad fuerte, hermanos míos, si no, yo aquí me quedaré.

Se van y su madre les pregunta dónde está Constandís.

- A nuestro Costandís lo casamos en Málgara,

le dimos una chica, madre, de doce años.

Por el contrario, en otras ocasiones son las canciones de Jaros las que han sido consideradas como acríticas. Así ocurre con las variantes conocidas como Apuesta

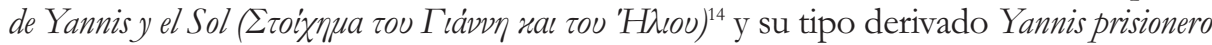

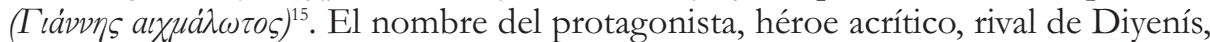
su identificación con el hijo de Andrónico ${ }^{16}$ y el carácter sobrenatural de sus hazañas han llevado a algunos estudiosos a incluirlas dentro de las canciones acríticas ${ }^{17}$. Sin embargo, el mito de la apuesta y el modo de presentación de la lucha no se corresponde con esta clasificación.

Por otra parte, muchos motivos comparten las composiciones acríticas con las fúnebres. Entre ellos destaca el del imposible rescate ofrecido a Jaros por el propio difunto o por su familiares. Esta tradición, con orígenes homéricos ${ }^{18}$, continúa en las composiciones acríticas pontias sobre la muerte de Diyenís, en las que el héroe intenta escapar a su destino ofreciendo a la personificación de la muerte, a cambio de su vida, dinero, sus armas e incluso su caballo ${ }^{19}$. Sin embargo, en los miroloyia

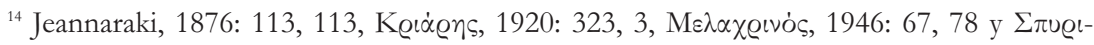

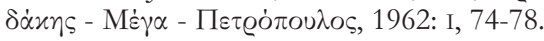

${ }^{15}$ Bajo estas dos denominaciones se incluye un número bastante amplio de textos (103 variantes en 1983 en los registros del KEE $\Lambda$ ), del que solo las formas extremas, realmente inconexas entre sí, se corresponden con estos títulos. Existen situaciones intermedias, en las que la presencia o la ausencia del motivo de la apuesta es lo que determina si un texto pertenece a una u otra categoría. Los dos grupos tienen diferente reparto geográfico e importancia numérica. El motivo de la disputa existe solo en catorce variantes, de las que doce tienen su origen en el Egeo y en las regiones costeras. Las del segundo tipo, aunque se atestiguan ampliamente en esa zona, en su mayoría aparecen en Grecia continental. En Tracia se registraron once, $c$. Saunier, 2001: 153-176. Se presenta ya en las primeras reco-

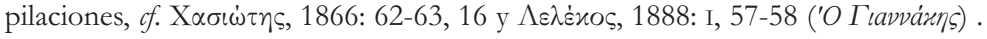

${ }^{16}$ Parece tratarse de una recreación de Andrónico Ducas, padre de Constantino Ducas, que vivió durante el reinado de León VI el Sabio. $C f$. una semblanza sobre su vida y hazañas en Ayensa

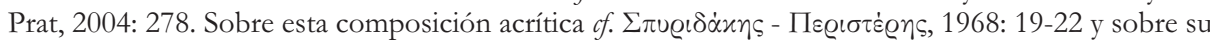

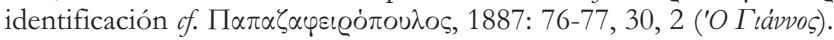

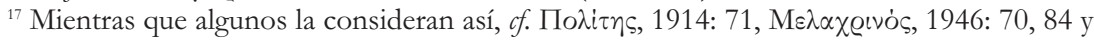

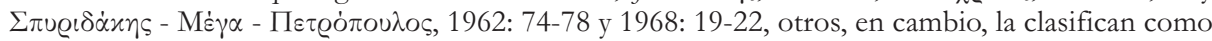
kléftica, $c f$. Passow, 1860: 124-125, CLXIV (Пavo. 64) (Adrianópolis) y 127, CLXIX, $\Lambda \varepsilon \lambda \dot{\varkappa} \varkappa o \varsigma, ~ 1888:$ II, 57

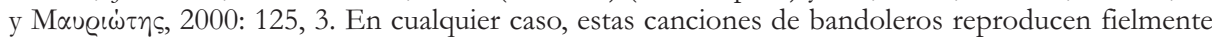
los antiguos esquemas acríticos.

${ }^{18} \mathrm{Il}$. I, VI, XI, XXIII y especialmente XXIV.

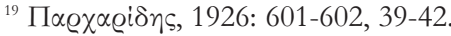


la guerra ha sido sustituida por la piratería, especialmente proveniente del mar, como evidencia el empleo de un léxico relativo.

Otra forma del propio mito del rescate se presenta mediante la petición del muerto a sus padres de que ofrezcan regalos a Jaros y a su familia, para que le permitan volver a su casa por las grandes fiestas. Este motivo se ha desarrollado especialmente en el Peloponeso ${ }^{20}$, pero no está ausente de Tracia, aunque, en esta

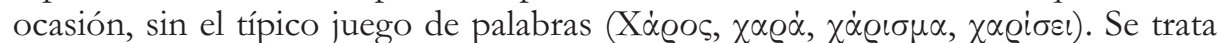
de un miroloyi dialogado, entre una chica joven y sus progenitores, procedente de Tsandó (Selimbria).

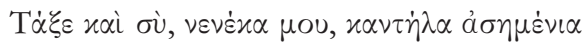

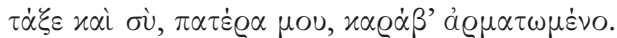

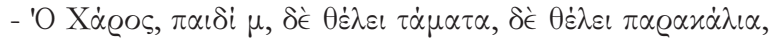

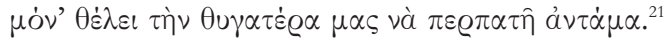

Prométele tú, madre mía, una lamparilla plateada y tú, padre mío, prométele una nave bien aparejada.

- Jaros, niña mía, no quiere ofrendas, no quiere súplicas, sino que quiere a nuestra hija que vaya con él.

Sin embargo, las canciones de Jaros son las que participan de más situaciones típicas con las acríticas. Así, en la composición Jaros y el pastor ('O Xáeos rai ó Booxós) se hallan el lugar de encuentro de los dos protagonistas ${ }^{22}$ y las referencias a la lucha-localización (era de mármol ${ }^{23}$, de bronce ${ }^{24} \mathrm{o}$ de hierro ${ }^{25}$ ), duración ${ }^{26} \mathrm{y}$

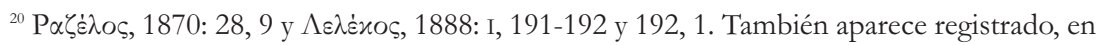

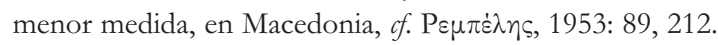

${ }^{21} \sum \tau \alpha \mu \circ \dot{\lambda} \lambda \eta \sum \alpha \varrho \alpha \nu \tau \dot{\eta}, 1929: 135,8$. Cf. otra variante oriental idéntica procedente de Tiroloi en $\sum \tau \alpha \mu o \dot{\lambda} \lambda \eta \sum \alpha \varrho \alpha \nu \dot{\eta}, 1939: 200,339$.

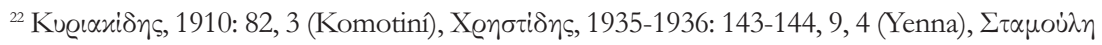

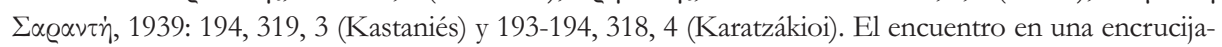

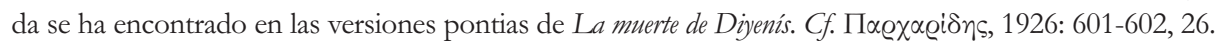

${ }^{23}$ Passow, 1860: 307-308, ccccxxxiI, 7-8 (Blau et Schlottmann Berichte d. A. D. W. Berl 1855,

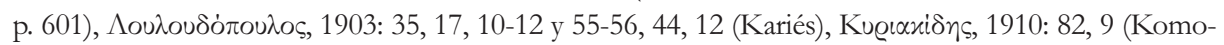

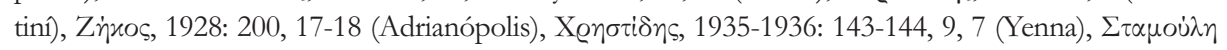

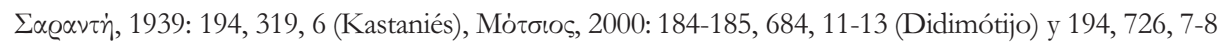
(Samotracia). Para las canciones acríticas of. una variante de la canción La lucha entre Tsamadó y su bijo

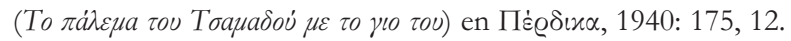

${ }^{24}$ Emplazamiento propio de las canciones del Ponto.

${ }^{25}$ Jeannaraki, 1876: 142-143, 142, 13 y 214, 276, 12 (Creta).

${ }^{26}$ Cf. una variante cretense de la canción Combate de Diyenís o de Yannis con un dragón en Jeannaraki, 1876: 98, 78, 7 . 
consecuencias de la misma ${ }^{27}$. Sin embargo, no todos los investigadores aceptan la hipótesis de la influencia de las canciones del ciclo acrítico, así G. Saunier propone la existencia de motivos comunes a ambos ciclos desde la base de una mutua independencia ${ }^{28}$.

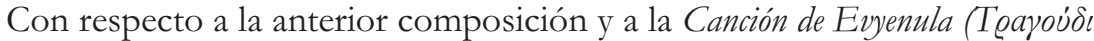
$\tau \eta \varsigma$ Euycvoúlas), un motivo compartido con la tradición acrítica sería la alusión a la tienda de $\operatorname{Jaros}^{29}$.

Realmente esta última composición parece tratarse de una variante del tipo segundo de la canción acrítica, derivada como canción de Jaros, titulada La prometida de Costandís, conocida en muchas partes de Grecia, desde Capadocia hasta el Heptaneso ${ }^{30}$, que desarrolla el motivo de la grave enfermedad y de la muerte de uno de los prometidos y del consiguiente suicidio del otro. Aquí la que enferma es la prometida de Costandís —Evyenula en la mayoría de las variantes ${ }^{31}$ — , que, en algunas versiones, cuando se entera de que se acercan los familiares de su prometido, llega incluso a dar la orden a su madre de que no les diga nada y vista de novia a su hermana pequeña ${ }^{32}$.

Común a estas canciones es también el motivo del agarre por los cabe$\operatorname{llos}^{33}$, que se ha encontrado, con una mínima variación, en la composición acrítica La doncella valiente ${ }^{34}$. Esta acción se atribuye ahora a un turco enamorado. Sirvan de ejemplo dos composiciones tracias — San Jorge ('O 'Aï $\Gamma \varepsilon \dot{\varepsilon} \varrho y \eta \varsigma)$ se titula la última de ellas- provenientes de Adrianópolis, cuyo verso es idéntico en ambas.

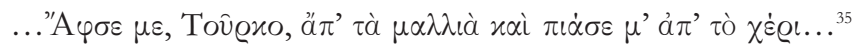

${ }^{27}$ Passow, 1860: 307-308, ccccxxxiI (Blau et Schlottmann Bericbte d. A. D. W. Berl 1855, p. 601),

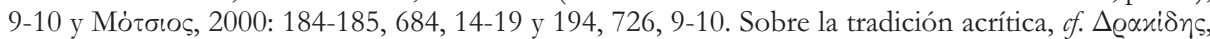

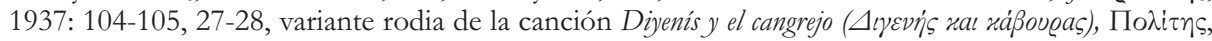
1909: 207-210, 1, 19-21 y 216-218, 5, 51-52, versiones chipriota y rodia de La muerte de Diyenís [cf.

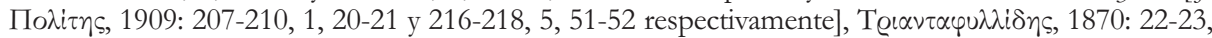
46-47, variante del Ponto de El hijo de Andrónico (Tou yrou tou Avboovirou) y de La lucha entre Tsamadó y su

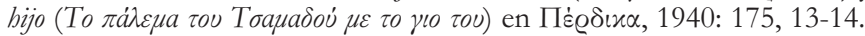

${ }^{28}$ Saunier, 2001: 296-310.

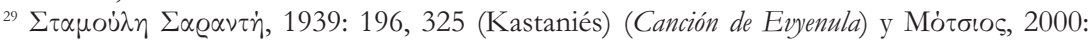
194, 726 (Canción de Jaros y el pastor). Sobre la tradición acrítica of. una variante de Quíos de la muerte

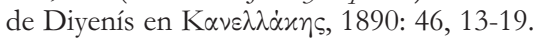

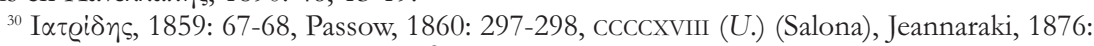

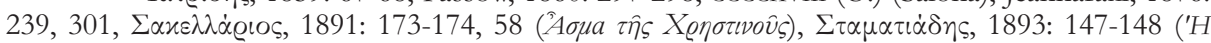
yróon rai ó Xáoos) y Koidens, 1920: 337, 2.

${ }^{31}$ Lüdeke, 1943-1947: 216, 145 (Chipre).

${ }^{32}$ Dos variantes tracias de este tipo, clasificadas como canciones de boda, procedentes de

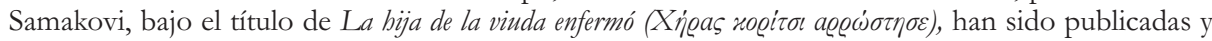

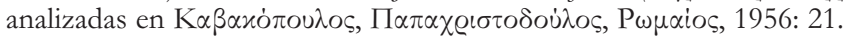

${ }^{33}$ Passow, 1860: 307-308, CCCCXXXII (Blau et Scblottmann Bericbte d. A. D. W. Berl 1855, p. 601),

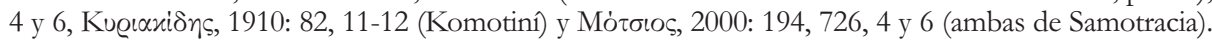

${ }^{34} C f$. una variante de Mikonos en Ayensa Prat, 2004: 58.

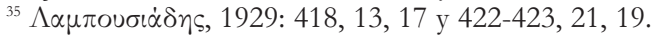




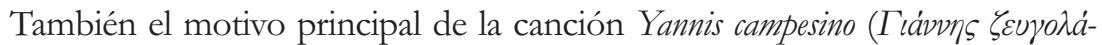
$\tau \eta \varsigma$ ), el arado y el anuncio de los pájaros, tiene igualmente origen acrítico [El rapto

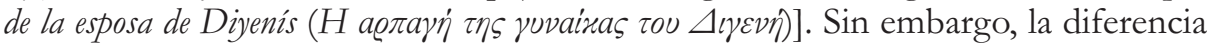
radica en el contenido del canto: en la forma originaria le comunica el rapto de su mujer, en la otra, en cambio, le predice su muerte. No obstante, esta última noticia proviene de las variantes del Ponto sobre la muerte del Acritas. Precisamente esta idea del engaño de los pájaros ( $\left(\mathrm{M} \varepsilon \gamma_{\varepsilon} \lambda \dot{\alpha} \sigma \alpha v \varepsilon \tau \alpha \pi 0 u \lambda(\dot{\alpha} »)\right.$ ) se ha registrado en los miroloyia tracios ${ }^{36}$.

Por último, abundantes son los versos formularios que remontan a la tradición acrítica. Muchos se encuentran en las canciones de Jaros, así en una variante de la Canción de Evyenula de Ebro aparecen dos fórmulas comunes. La primera, registrada también en la Canción de Jaros y el pastor ${ }^{37}$, se refiere al enfado de la personificación de la muerte ante las palabras altivas de los protagonistas.

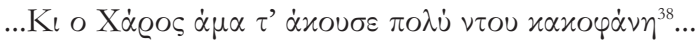

La fórmula se halla atestiguada en numerosas composiciones acríticas, entre otras en una versión procedente de Episkopí (Pafos) de la canción Diyenís y la bija

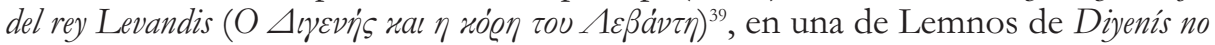

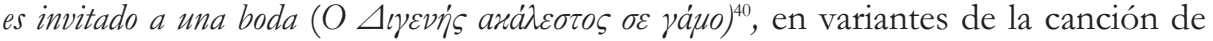

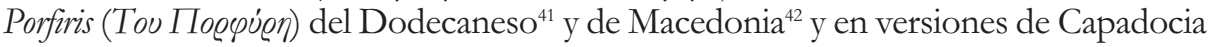
de Los hijos de Andrónico (o de Diyenis) y el fantasma ${ }^{43}$ y de La doncella valiente ${ }^{44}$.

La segunda fórmula se refiere al poder de la familia de la joven, hermanos y marido:

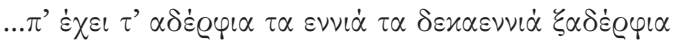

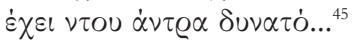

Recuerdan estas palabras las puestas en boca de la esposa del héroe en una

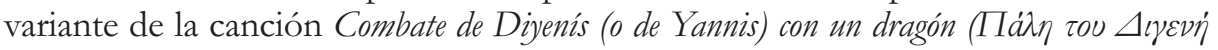

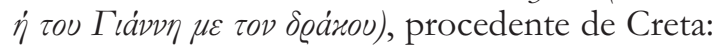

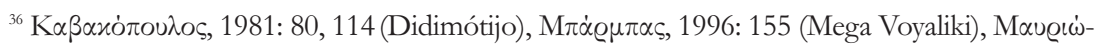

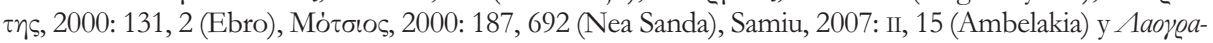

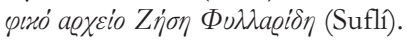

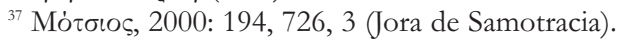

${ }^{38}$ Maveí่tns, 2000: 185, 5.

${ }^{39}$ Ku@ıаxions, 1926: 140-149, 94 у 106.

${ }^{40}$ KEE $\Lambda$ 1160, III, 17, 10 (1938).

${ }^{41}$ Baud-Bovy, 1935: I, 83-84, 9.

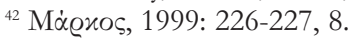

${ }^{43}$ KEE $\Lambda$ 228, 15, 12, 9.

${ }^{44}$ Lagarde, 1886: 36, 38, 22.

${ }^{45}$ Mav@í่тท, 2000:, 185, 3-4. 


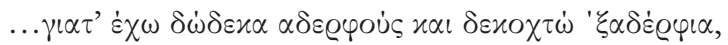

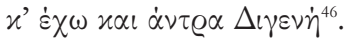

Igualmente los versos referentes al carácter humano del habla de los pájaros, portadores de malas noticias, que se registran en una variante de Kariés de la composición titulada Yannis campesino, aparecen también en otra canción acrítica de Tracia

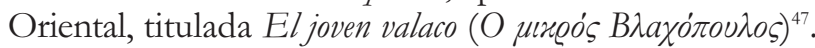

Con respecto a los lamentos fúnebres el inicio de carácter formulario del

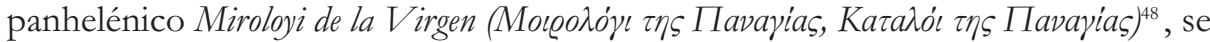
encuentra también en la tradición acrítica ${ }^{49}$. En esta ocasión se transcribe el de una variante procedente de Pirgos.

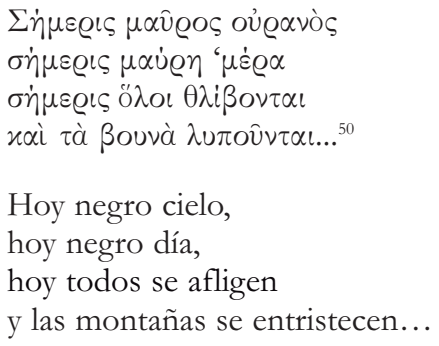

Igualmente ocurre con los versos iniciales, que enmarcan temporalmente

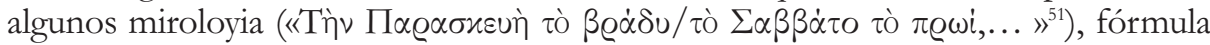
que se repite con ligeros cambios en canciones de otras categorías ${ }^{52}$ y que se remonta a la tradición acrítica ${ }^{53}$.

Pero no siempre las fórmulas se mantienen inalterables, sino que sufren modificaciones, adaptándose a las nuevas circunstancias. Así ocurre con una con la que suelen comenzar algunos trenos normalmente en forma de dísticos rimados ( $\Delta \Delta \grave{\epsilon} \nu$

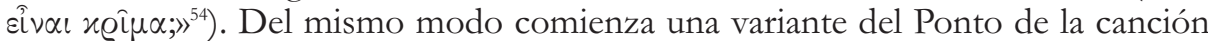
acrítica Jandinós $(O \Xi a ́ v \tau \iota v o v)$ :

${ }^{46} \mathrm{KEE} \Lambda, 428,250,5-6$.

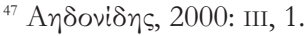

${ }^{48}$ Se trata de un extenso poema narrativo, en decapentasílabos yámbicos con rima, que goza de una gran aceptación popular. Inspirado en los episodios relativos de los Evangelios y en la himnografía de la iglesia, constituye un treno sobre la pasión de Cristo, visto a través de los ojos y de los sentimientos de su madre.

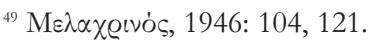

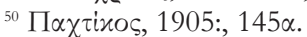

${ }^{51}$ Xenбiions, 1935-1936: 145, 13, 1-2 (Yenna).

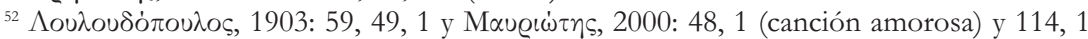
(canción histórica).

${ }^{53}$ KEE $\Lambda$ 1153, I, 11, 13, 1 (Beocia).

${ }^{54} \sum \tau \alpha \mu о \dot{\lambda} \lambda \eta \sum \alpha \varrho \alpha \nu \tau \dot{\eta}, 1939: 201,345,10$ (Selimbria). 


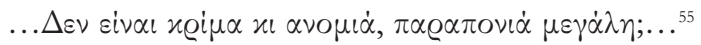

Por otra parte, la expresión, propia de los plantos para referirse a Jaros

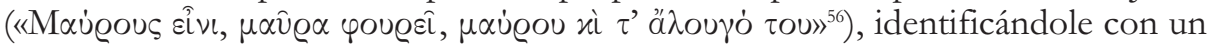
negro jinete, aparece atestiguada en una variante de la canción acrítica Andrónico

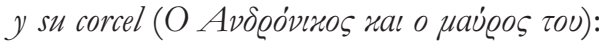

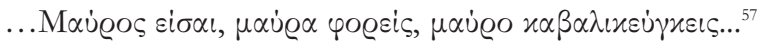

Con variaciones también aparece en algunas versiones chipriotas de La muerte de Diyenis ${ }^{58}$.

Puede concluirse afirmando que los lazos entre las canciones acríticas y las de Jaros son mucho más estrechos, consecuencia de que ambas categorías abordan una temática similar en torno a la lucha del héroe, entrecruzando así estas temas y motivos de aquellas. Sin embargo, los miroloyia también participan, aunque en menor medida, de temas y motivos comunes y del abundante y rico acervo formulario, propio de toda la poesía popular griega. Aunque en el presente estudio se han analizado especialmente composiciones populares de Tracia, sus conclusiones pueden extrapolarse también al resto del territorio helénico, ya que la región no presenta diferencias significativas en el tratamiento de estos temas.

\section{REFERENCIAS BIBLIOGRÁFICAS}

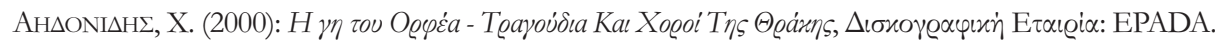
Alexiou, M. (1974): The ritual lament in Greek tradition, Cambridge.

Ayensa Prat, E. (2004): Cancionero griego de frontera, Madrid.

Baud-Bovy, S. (1936): La chanson populaire grecque du Dodécanèse, I. Les textes, París.

Danforth, L. - Tsiaras, A. (1982): The Death Rituals of Rural Greece, Princeton.

$\triangle$ PAKI

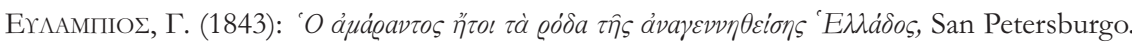

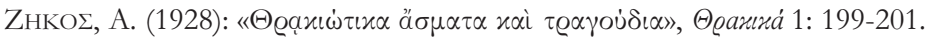

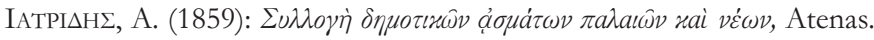

JeAnNARAKI, A. (1876 [2010]): Kretas Volkslieder Nebst Distichen und Sprichwortern. In der Ursprache mit Glossar, Leipzig.

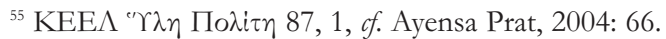

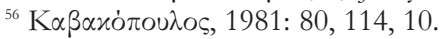

${ }^{57}$ M $\alpha \nu \omega \lambda \alpha x \dot{\alpha} x \eta \varsigma, 1896: 220,2,6$.

${ }^{58}$ Пoגiins, 1909: 2017-210, 1,1. 


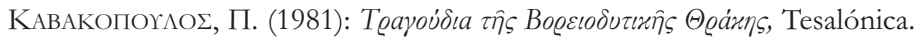

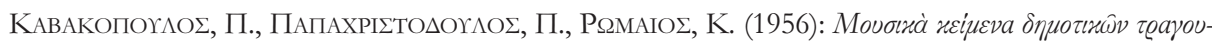

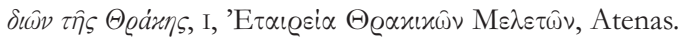

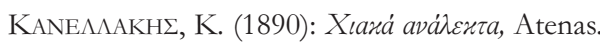

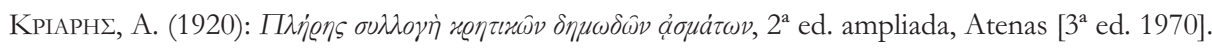

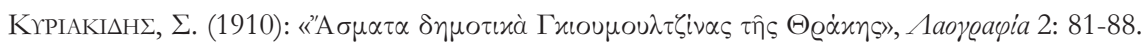

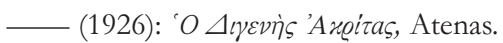

Lagarde, P. DE (1886): Neugrieschisches aus Klein Asien, Gotinga.

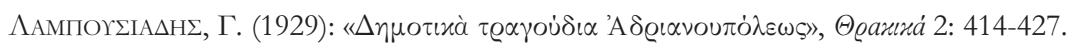

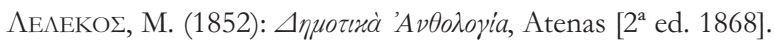

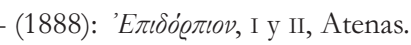

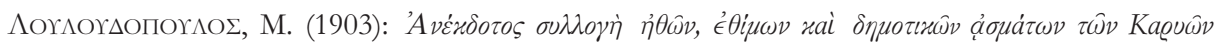

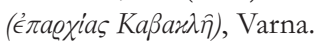

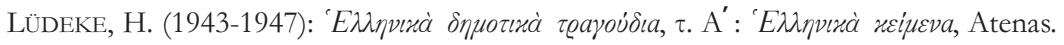

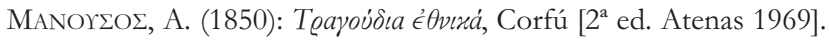

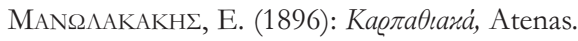

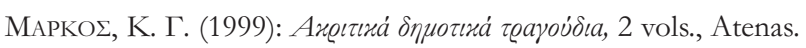

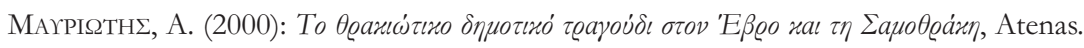

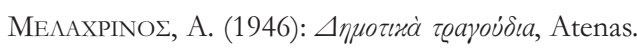

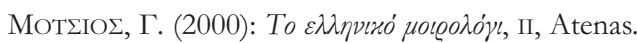

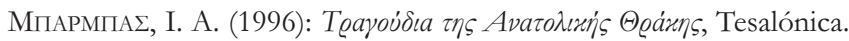

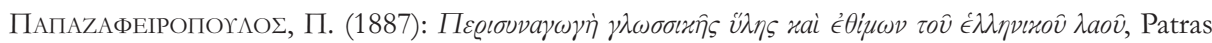
[2 ed. D. N. Caravías, Atenas, 1977].

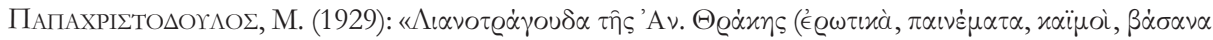

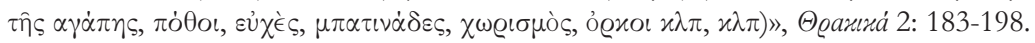

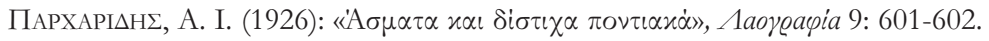

PAssow, A. (1860): Popularia carmina Graeciae recentioris, Leipzig [2 $2^{\mathrm{a}}$ ed. Atenas, 1958].

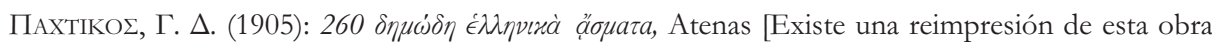
hecha en Atenas por D. N. Caravías en 1992].

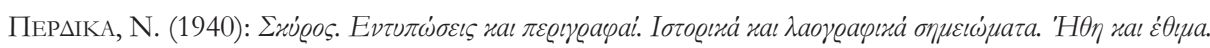

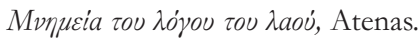

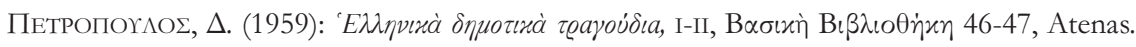

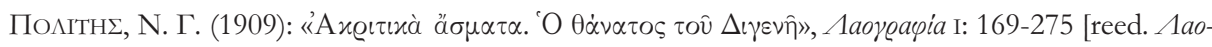

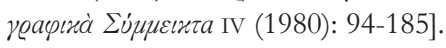

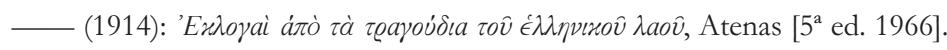

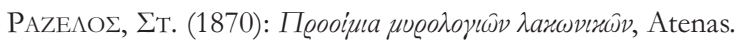

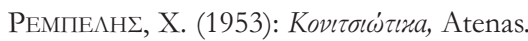

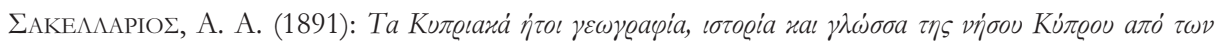

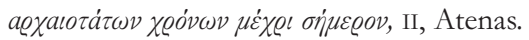




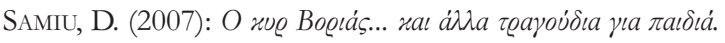

Saunier, G. (1989): «La fille guerrière et la trahison du saint», Mètis. Anthropologie des mondes grecs anciens IV: 61-85.

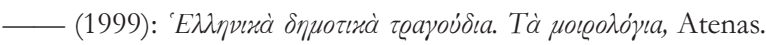

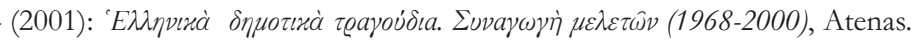

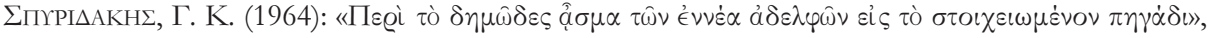
EAA 15-16: 3-13.

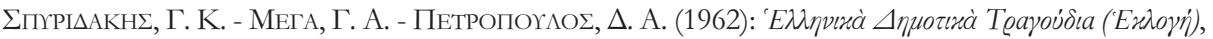

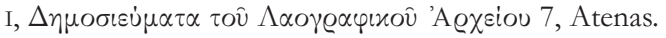

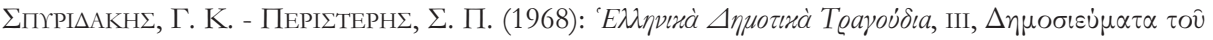

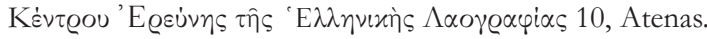

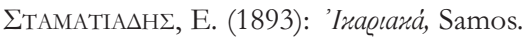

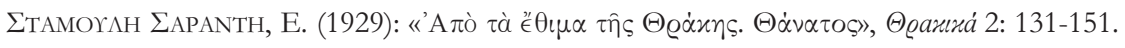

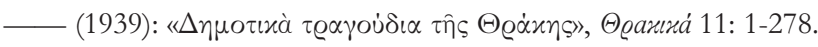

Tommaseo, N. (1842): Canti popolari toscani, corsici, illirici, greci, III, Venecia.

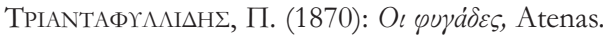

VAn Gennep, A. (1909): Les rites de passage, París [Los ritos de paso, trad. de Juan Aranzadi, Alianza Editorial, Madrid 2008].

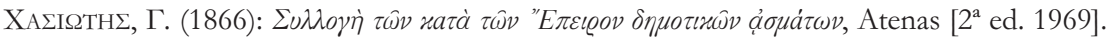

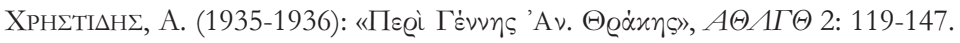

\section{SigLAS UTILIZADAS}

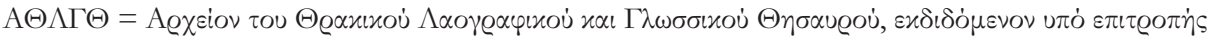

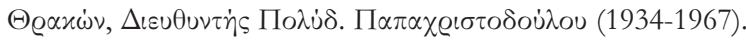

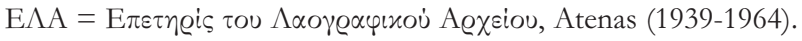

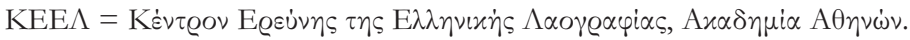

